\title{
Diacronie
}

Studi di Storia Contemporanea

$N^{\circ} 8,4 \mid 2011$

Le sembianze di Clio: approcci alla storia

\section{Massimo Pistacchi (a cura di), Vive Voci. L'intervista come fonte di documentazione}

Jacopo Bassi e Alessandro Cattunar

\section{(e) OpenEdition}

Edizione digitale

URL: http://journals.openedition.org/diacronie/3484

DOI: 10.4000/diacronie.3484

ISSN: 2038-0925

Editore

Association culturelle Diacronie

Notizia bibliografica digitale

Jacopo Bassi e Alessandro Cattunar, " Massimo Pistacchi (a cura di), Vive Voci. L'intervista come fonte di documentazione », Diacronie [Online], № 8, 4 | 2011, documento 8, Messo online il 29 octobre 2011. consultato il 23 septembre 2020. URL : http://journals.openedition.org/diacronie/3484; DOI : https:// doi.org/10.4000/diacronie.3484 


\section{RECENSIONE:}

\section{Massimo PISTACCHI (a cura di), Vive Voci. L'intervista come fonte di documentazione, Roma, Donzelli, 2011, 207 pp.}

a cura di Jacopo BASSI, Alessandro CATTUNAR *

Vive voci è la raccolta degli interventi discussi in occasione del convegno, svoltosi dal 5 al 7 maggio 2009 presso la Biblioteca nazionale di Roma, dedicato a L'intervista come «fonte» di documentazione. Curatore di questa raccolta di saggi è Massimo Pistacchi, direttore dell'Istituto centrale per i beni sonori ed audiovisivi. Alla base della realizzazione di queste giornate di studio vi era la volontà di riflettere sulla tematica delle fonti orali, della loro raccolta e della loro trattazione. A distanza di ventitre anni dal grande convegno realizzato nel maggio del 1986 - L'intervista strumento di documentazione. Giornalismo, antropologia, storia orale - che vide la partecipazione di autorevoli contributori (i giornalisti Giovanni Ferrara, Gianni Letta, Walter Mauro, Gianni Minà, Enzo Roggi; i sociologi Diego Carpitella, Domenico De Masi, Danilo Dolci, Nuto Revelli, Luigi Lombardi Satriani; gli storici Gabriele De Rosa, Giuseppe Galasso, Paolo Spriano, Lucio Villari) i tempi erano maturi per una nuova analisi della fonte orale, anche alla luce dei mutamenti intervenuti nel campo delle tecnologie disponibili per realizzare le registrazioni, ma soprattutto in considerazione dell'affermarsi di una nuova sensibilità storica che considera oggi, unanimemente, tali documentazioni come fonti storiche tout court.

Come in occasione del convegno del 1986 l'approccio allo strumento di indagine e di lavoro rappresentato dall'intervista è stato affrontato da specialisti operanti in ambiti di ricerca e professionali diversi: antropologia, archivistica, giornalismo, sociologia e storia. Attraverso la lettura dei singoli contributi si può constatare come in questi anni 
si sia approdati alla condivisione - anche in diversi ambiti disciplinari - di alcuni indirizzi metodologici fondamentali.

Nella premessa Massimo Pistacchi ricorda come sia in corso una progressiva affermazione dell'importanza degli archivi sonori e audiovisivi; la riflessione si sposta poi sul senso e i mutamenti occorsi nel campo delle tecnologie utilizzate nella fase realizzativa delle interviste e sull'affidabilità e la qualità delle registrazioni. Lo stesso autore evidenzia come siano ancora molte le carenze, in Italia, nell'utilizzo delle fonti orali come fonte di documentazione, carenze legate soprattutto alla mancanza di standard comuni e condivisi per la realizzazione delle stesse e per una catalogazione sistematica.

L’intervento di Antonella Fischetti e Piero Cavallari parte proprio da alcune riflessioni sul convegno svoltosi nel maggio del 1986 e su quanto emerso in quell'occasione: al posto di una concezione che rivendica la trasparenza del ricercatore, che dovrebbe apparire il più possibile neutrale rispetto alle affermazioni dell'intervistato - idea già sconfessata da Portelli nel convegno del 1986 - si propone un nuovo approccio, più attento all'aspetto dell'“intersoggettività", della relazione tra tutti gli attori che prendono parte ad un'intervista, sottolineando in particolare l'importanza degli interventi del ricercatore. L’intervistato assume così connotati nuovi, non definiti aprioristicamente dall'attitudine assunta da colui che intervista.

Alessandro Portelli, subito dopo, rivendica e sottolinea la differenza fra l'intervista in sé - intesa come evento, incontro, esperienza - e la registrazione, documento utilizzato e utilizzabile anche da terzi. Ribadire il fatto che l'intervista è un documento creato volontariamente dal ricercatore appare fondamentale da un punto di vista metodologico. Viene poi sottolineata la dimensione essenzialmente narrativa della fonte orale, in cui la relazione tra ricercatore e testimone deve essere tesa sostanzialmente a far emergere la soggettività di quest'ultimo, le sue costruzioni identitarie e il modo di trasmetterle attraverso usi specifici della lingua e delle strutture del racconto.

Il contributo di Giovanni Contini verte sull'esperienza concreta della Soprintendenza Archivistica della Toscana nell'organizzazione di ricerche di storia orale nella regione. L'enorme patrimonio raccolto si è dovuto confrontare con gli ostacoli rappresentati dai problemi di legislazione - la normativa posta a tutela della privacy pone delle limitazioni all'uso di interviste che diano giudizi o menzionino persone ancora in vita o che abbiano parenti stretti che ne tutelino la memoria - e di fruizione dello stesso: il deposito della documentazione presso le biblioteche locali 
presenta infatti alcune criticità che gli studiosi devono considerare ancora prima di cominciare l'attività "sul campo".

Sara Zanisi ha voluto rievocare il ruolo pionieristico di Duccio Bigazzi nell'inserire la storia orale e l'intervista tra gli strumenti di lavoro della storiografia dell'industria italiana. In particolare sono prese in considerazione le ricerche svolte nello stabilimento Alfa Romeo-Portello tra lavoratori e manager nel periodo 1980-1995; in queste emerge la cifra metodologica utilizzata da Bigazzi nella sua attività: l'intervista autobiografica intesa, ed analizzata, in quanto "storia di vita".

Gli interventi di Dino Pesole, Marino Sinibaldi e Dario Laruffa sono invece dedicati alla riflessione sul senso e l'importanza dell'intervista giornalistica - spesso deformata e avvilita da una cattiva interpretazione della professione di giornalista - in un regime democratico e sulle necessità, per l'intervistatore, di curare "l'arte di ascoltare".

Pietro Clemente, invece, mette in evidenza le specificità dell'intervista antropologica a partire da quello che è stato il modello per il suo modus operandi: Ernesto de Martino. L'intervista per l'antropologo, ancora prima che per lo storico, appare come uno strumento imprescindibile di ricerca: «Nelle mie ricerche l'intervista è stata la forma fondamentale della conoscenza, dell'accumulazione del sapere, dell'esercizio di dialogo»1. Clemente si sofferma sull'evidente disagio che viene a prodursi nell'antropologo fra il dovere etico dell'intervistatore di raccontare la storia collettiva e la necessità di proteggere i protagonisti dei racconti (e i loro discendenti).

Anche l'intervento di Elena Bacchiddu si concentra sull'ambito etno-antropologico prendendo in esame sia la letteratura internazionale che quella italiana. Le strategie retoriche e gli espedienti linguistici che caratterizzano i racconti dei testimoni vengono qui descritti come «luogo centrale di costruzione delle realtà culturali e della loro rappresentazione $»^{2}$.

Il saggio di Martina Giuffrè e Valentina Lapiccirella Zingari riflette sulle ricerche di tipo qualitativo basate sui racconti di vita evidenziando l'importanza di cogliere ciò che non è esplicitamente contenuto nel testo: contesti, relazioni, situazioni. L'intervista produce quindi «effetti sorprendenti e, pensata come "fonte rivitalizzante", guida la riflessione ad una considerazione delle pratiche etnografiche come luoghi d'ascolto creativo»3; l'elaborazione delle interviste, quando viene operata dal ricercatore in

\footnotetext{
${ }^{1}$ CLEMENTE, Pietro, L'antropologo che intervista. Le storie della vita in PISTACCHI, Massimo (a cura di), Vive Voci. L'intervista come fonte di documentazione, Roma, Donzelli, p. 84.

2 BACCHIDDU, Elena, Scrivere, leggere, rappresentare $i$ discorsi orali. Testualizzazione, edizione in Ibidem, p. 90.

3 GIUFFRÈ, Martina, LAPICCIRELLA ZINGARI, Valentina, Oltre il testo, oltre l'intervista. Sguardi etnografici in Ibidem, p. 139.
} 
stretta collaborazione con i responsabili delle istituzioni museali - come dimostra l'esperienza di Modane - va proprio in questa direzione.

Maria Immacolata Macioti osserva come l'intervista sociologica consenta riflessioni, non solo metodologiche ma anche teoriche, sugli obiettivi stessi della ricerca che si sta compiendo. L'autrice si sofferma poi ad analizzare le tematiche e le problematiche concrete che emergono nel corso della realizzazione di un'intervista: le difficoltà linguistiche, le reticenze, la trascrizione.

Il contributo di Roberto Cipriani si sofferma sull'utilità di alcuni software - e dell’informatica in termini più generali - per la gestione dei dati e dei risultati delle interviste quantitative e qualitative.

Franco Ferrarotti, infine, rivendica la «presenza del corpo» dell'intervistato come una necessità dell'intervista sociologica e muove alcune osservazioni in merito alla differente concezione dell'intervista in sociologia e in storia; l'autore osserva infatti come le garanzie di precisione e attendibilità - possibile punto di partenza per una possibile integrazione fra le due discipline - siano contenute proprio nel rigore metodologico impiegato per le ricerche.

Il complesso dei saggi offre dunque una visione d'insieme dello status dell'intervista in diversi campi delle scienze umane. L’impressione generale che si ricava è quella di un sostanziale accordo - sia da un punto di vista teorico e analitico, sia sul versante tecnico-metodologico - all'interno delle ricerche accademiche riferibili alle scienze sociali, ma anche del permanere di una sostanziale distanza interpretativa con l'ambito giornalistico, in cui l'intervista viene intesa forse più come uno strumento che come un documento. Gli interventi dei giornalisti evidenziano una distanza non solo terminologica rispetto a quelli concepiti in ambito accademico: più che sulla metodologia - vero e proprio leitmotiv comune a sociologi, antropologi e storici questi sono imperniati sull'etica e rievocano i richiami schienadrittisti della Presidenza della Repubblica.

Quanto evocato in relazione alla necessità di spogliarsi di preconcetti e conoscenze pregresse nel momento in cui un ricercatore decide di attivare una relazione di scambio (un inter-vista appunto) con un testimone, appare radicalmente confutato dalla visione giornalistica: « [...] l’intervista molto spesso costituisce per il giornalista uno strumento di grande importanza per acquisire informazioni, elementi di back-ground che poi possono essere utilizzati in successivi articoli o approfondimenti analitici. Per questo, la preparazione è fondamentale. Occorre documentarsi, aggiornarsi fin nei minimi 
particolari, entrare in possesso cioè di tutti gli elementi di coscienza necessari per "dominare" l'argomento»4.

L'intervista come fonte di documentazione ha dunque assunto due declinazioni: quella più funzionale e didascalica, propria della professione di giornalista, che deve guardare alle ragioni deontologiche di equità e libertà dell’informazione, e quella inerente alle scienze umane - più attenta ai suo aspetti linguistici e narrativi, alla sua contestualizzazione nella società, che necessita di una chiara metodologia e impone di mantenere ben chiara la distinzione fra l'intervista-evento e l'intervista-documento.

4 PESOLE, Dino, L’intervista, uno strumento chiave per il diritto all'informazione in Ibidem, $\mathrm{p}$. 46. 


\section{* Gli autori}

Jacopo Bassi è dottore magistrale in Storia d'Europa. Si è laureato in Storia della Chiesa presso l'Università di Bologna nel 2008; si è occupa di identità e religione nel sudest europeo. Attualmente collabora con la casa editrice Il Mulino.

URL: < http://www.studistorici.com/2009/02/24/jacopo_bassi >

Alessandro Cattunar è dottorando in Storia Contemporanea presso l'Istituto italiano di Scienze Umane - SUM (Firenze, Napoli). Già dottore magistrale in Storia d'Europa (Bologna 2008) e specializzato in Media Education (Università Cattolica di Milano 2008), presiede l'Associazione Quarantasettezeroquattro (Gorizia) e collabora con l'Associazione Italiana di Storia Orale, l'Istituto Friulano per la Storia del Movimento di Liberazione e l'Istituto storico di Torino. Si occupa dei rapporti tra media e storia e di quelli fra memoria e identità.

URL: < http://www.studistorici.com/2008/o9/14/alessandro_cattunar >

\section{Per citare questo articolo:}

BASSI, Jacopo, CATTUNAR, Alessandro, «Recensione: Massimo PISTACCHI (a cura di), Vive Voci. L'intervista come fonte di documentazione, Roma, Donzelli, 2011, 207 pp.», Diacronie. Studi di Storia Contemporanea: Le sembianze di Clio: approcci alla storia, 29/10/2011,

URL:<http://www.studistorici.com/2011/10/29/bassi-cattunar_numero_8/ >

Diacronie Studi di Storia Contemporanea $\beta$ www.diacronie.it

Risorsa digitale indipendente a carattere storiografico. Uscita trimestrale. redazione.diacronie@hotmail.it

Comitato di redazione: Marco Abram - Giampaolo Amodei - Jacopo Bassi - Luca Bufarale - Alessandro Cattunar - Alice De Rensis Barbara Galimberti - Deborah Paci - Fausto Pietrancosta - Martina Sanna - Matteo Tomasoni - Luca Zuccolo

Diritti: gli articoli di Diacronie. Studi di Storia Contemporanea sono pubblicati sotto licenza Creative Commons 2.5 Possono essere riprodotti a patto di non modificarne i contenuti e di non usarli per fini commerciali. La citazione di estratti è comunque sempre autorizzata, nei limiti previsti dalla legge. 